\section{Cahiers de Narratologie}

Analyse et théorie narratives

31 | 2016

Sérialité narrative. Enjeux esthétiques et économiques

\title{
Logique imaginaire et logique de marché : analyse d'une création de fans dans le jeu vidéo
}

Le cas de España en llamas sur Call of Duty 2

\section{Marc Marti}

\section{(2) OpenEdition}

\section{Journals}

Electronic version

URL: http://journals.openedition.org/narratologie/7547

DOI: 10.4000/narratologie.7547

ISSN: 1765-307X

\section{Publisher}

LIRCES

\section{Electronic reference}

Marc Marti, «Logique imaginaire et logique de marché : analyse d'une création de fans dans le jeu vidéo », Cahiers de Narratologie [Online], 31 | 2016, Online since 22 December 2016, connection on 15 November 2019. URL : http://journals.openedition.org/narratologie/7547

This text was automatically generated on 15 November 2019.

Article L.111-1 du Code de la propriété intellectuelle. 


\title{
Logique imaginaire et logique de marché : analyse d'une création de fans dans le jeu vidéo
}

Le cas de España en llamas sur Call of Duty 2

\author{
Marc Marti
}

1 Par sa nature, le jeu vidéo a souvent été considéré comme un pur produit industriel, soumis uniquement aux lois du marché et affichant donc des caractères irréductibles à l'idéologie esthétique traditionnelle, qui lui attribue par ailleurs un caractère de futilité, provenant essentiellement de sa nature ludique. Si nous suivons cependant Jean-Marie Schaeffer, « la question de la fiction est aussi celle des jeux vidéos et de la cyberculture $^{1} » . \mathrm{Au}$ moment de s'interroger sur la notion de sérialité, le jeu vidéo mérite une attention particulière, car il relève de nouvelles pratiques contemporaines. Celles-ci se caractérisent par la multiplicité des supports sur lesquels le récit se développe, un phénomène qui contribue à modifier la notion de sérialité monomédiale au profit de la déclinaison sérielle transmédia. De fait, ce nouveau mode de récit sériel peut permettre d'étudier comment évoluent les rapports du public aux genres narratifs contemporains. En effet, le livre, le cinéma ou la télé, qui furent traditionnellement à la base de la création narrative sérielle sur média unique, se combinent maintenant avec le jeu vidéo, le film d'animation, la bande dessinée pour ne citer que les supports les plus fréquents.

2 Il s'agira pour nous de questionner d'abord la notion de sérialité non seulement à partir de sa valeur esthétique, mais aussi à partir des fonctions sociales qu'elle en vient à assumer, car comme nous le verrons, la sérialité est tout autant un mode de production des objets culturels qu'un mode de réception. Il conviendra en particulier d'insister sur le fait que la réception " peut mettre en série » des objets qui au départ n'avaient pas été conçus dans cette optique.

3 Ainsi, les nouvelles formes de la culture contemporaine ont fait émerger un autre type de sérialité, celle d'une déclinaison narrative dans le transmedia storytelling, qui place 
le spectateur dans une position créative, ou du moins dans un mode de consommation dynamique et non linéaire.

4 Nous tenterons pour finir de mettre en valeur la relation qui s'établit entre le public et la série, en examinant en particulier de quelle façon celle-ci peut conditionner une fan fiction, constituée dans le cadre de notre contribution par un greffon (mod) de jeu vidéo.

\section{De la série à la convergence culturelle}

5 La notion de sérialité semble aller de soi dans le cadre des pratiques traditionnelles de la littérature et de la culture audiovisuelle. Les travaux universitaires sur le roman feuilleton comme objet historique, esthétique et culturel sont nombreux. Les recherches relativement récentes sur les séries télé en constituent sans doute le pendant le plus contemporain. La sériation y est souvent pensée comme une pratique des « industries culturelles », qui (re)produit des récits de type feuilletonesque, selon un principe narratif de gestion temporelle qui reste constant. L'unité serait ainsi constituée par l'épisode et la série d'épisodes constituerait un ensemble fini. Ce schéma reste cependant théorique car les pratiques - tant de production que de consommation - sont plus complexes.

6 Le roman feuilleton reposait en effet sur une série d'épisodes dont le final ne résolvait jamais toute la tension narrative mise en place par l'intrigue. Une fois terminés, ces épisodes constituaient un roman. Un autre type de série est la série romanesque ou série de romans, en général écrite par un auteur unique ${ }^{2}$. Pour citer un cas connu en Espagne, Manuel Vázquez Montalbán a créé la série du détective Pepe Carvalho, qui a connu un très grand succès dans les années 1980 et 1990. L'ensemble est constitué par des œuvres autonomes, des personnages uniques et de nombreux personnages récurrents, dont le héros. Cependant, l'auteur les fait évoluer et vieillir au fur et à mesure des années. Le détective trentenaire de 1975 devient un sexagénaire dans les années 2000. Le dernier opus, écrit juste avant la mort du propre auteur, constitue un véritable testament. Le roman, en dehors des normes des précédents - bien plus long et publié en deux tomes - porte le titre Milenio (Millénaire). Il clôt la série en suggérant que le nouveau millénaire n'est plus adapté au détective, à moins que ce ne soit le contraire. On voit donc que du côté des créateurs, la série peut être un ensemble d'épisodes qui constitue une œuvre, mais aussi un ensemble d'œuvres qui constituent un monde.

7 L'industrie littéraire, dans son versant éditorial, a donné un sens plus extensif encore à la sérialité, en structurant ses catalogues selon des séries thématiques. Aux origines de ces séries, on retrouve d'abord des formes narratives récurrentes, tout comme les personnages et l'action. L'identification et la reconnaissance par les lecteurs de ces prototypes narratifs récurrents plus génériques en ont aussi fait une catégorie marketing. Chez l'éditeur, le genre littéraire est remplacé par la série, le roman policier devenant par exemple la série noire chez Gallimard. La sérialité en littérature est ainsi une notion qui recouvre des réalités assez larges, allant de la pratique créative feuilletonesque à la création de séries de romans de même genre (littéraire).

8 La série télévisée n'a pas réellement changé cette structuration qui fonctionne par épisodes avec final ou par épisodes engageant une suite. Pour Henri Jenkins, la structure feuilletonesque classique a été conservée jusque dans les années $1980^{3}$. Dans les années 1990, sensibles à l'apparition d'un public qui pouvait revoir les épisodes ou les visionner en différé grâce aux artéfacts technologiques, les nouveaux auteurs « ont 
eu tendance à privilégier des histoires se déroulant sur toute une saison (ainsi que des intrigues ou des informations qui se déployaient sur plusieurs saisons $\left.{ }^{4}\right) »$. Un prototype parlant de ce genre de série serait les X-Files. L'attitude du public devenait celle d'un spectateur engagé, qui avait fait l'effort de suivre les saisons. Le dernier tournant s'est amorcé dans les années 2000, avec des séries (Les Sopranos, The Shield, etc...) qui se caractérisent par un casting étoffé (nombreux personnages), «des histoires étendues, ainsi que par une intensification et un ajournement permanent de l'intrigue et du dénouement ${ }^{5} »$. Temporellement, ces séries proposent trois types d'unités : l'épisode, la saison, la collection complète.

9 Le cinéma est entré dans le système sériel très précocement. Les œuvres proposant la déclinaison du même personnage vivant différentes aventures fonctionnent sur ce principe, qui va toujours de pair avec un succès commercial. Le cinéma muet italien, qui a inventé le personnage de Maciste, l'a utilisé pendant une soixantaine d'années. La proposition d'œuvres sous forme de sagas a aussi favorisé la consolidation de ces productions.

Toutes ces sérialités classiques ont connu une dernière évolution, avec le passage des récits au transmedia storytelling, tel que le définit Henry Jenkins.

\section{De la sérialité au transmedia}

11 Cette conception de la sérialité, autant dans les pratiques de production que dans les pratiques de réception, a été amenée à évoluer. En effet, la création contemporaine est marquée par la multiplicité des supports sur lesquels le récit de fiction se développe. Le livre, la télé ou le cinéma, qui furent traditionnellement à la base de ce type de création, se combinent maintenant avec le jeu vidéo, le film d'animation, la bande dessinée pour ne citer que les supports les plus fréquents. Henry Jenkins définit le storytelling transmedia ainsi ${ }^{6}$ :

Dans la conception idéale du storytelling transmedia, chaque media fait ce qu'il sait faire le mieux : un récit peut ainsi être introduit dans un film, puis se développer à travers la télévision, le roman, la bande dessinée. Son univers peut être exposé au public par le jeu vidéo ou transformer en expérience vivante dans un parc d'attraction ou de loisirs. Chaque entrée de la franchise doit être indépendante, de sorte qu'il ne soit pas nécessaire de voir le film pour aimer le jeu vidéo, et vice versa. Tout produit donné est un point d'entrée dans l'ensemble de la franchise. La lecture transmedia permet une profondeur d'expérience qui incite toujours à davantage de consommation. La redondance et la répétition, en revanche, épuisent l'intérêt des fans et conduisent les franchises à l'échec.

Au bout du compte, le storytelling transmedia est devenu :

[...] l'art de construire un monde - car les artistes créent des environnements convaincants, qui ne peuvent être pleinement explorés ou épuisés dans une seule œuvre ou même un seul média.

On voit ainsi comment la sérialité traditionnelle a évolué avec cette pratique. D'une part, les séries sur un media unique, comme par exemple celle de la télévision, voient leur monde reproduit, sérié, sur d'autres supports. D'autre part, des œuvres " uniques ", qui semblent des objets esthétiques " non sériels ", se retrouvent dans des déclinaisons transmédiatiques, qui les font de fait entrer dans une forme particulière de sérialité, qui serait celle de la transmédialité. L'exemple du Blair Witch Project, développé par Henri Jenkins, illustre parfaitement ce dernier avatar de la sérialité7. 
Sans que le public "traditionnel», celui d'un seul média dominant (cinéma, télé, littérature) disparaisse, la pratique créative transmedia a aussi fait émerger un public plus hétérogène, correspondant à toutes les attitudes réceptives possibles. Céline Masoni-Lacroix propose une typologie de 9 niveaux, qui essaie de rendre compte de la gradation de ces attitudes de réception, en mesurant l'implication du public vis-à-vis des œuvres. ${ }^{8}$. Les deux derniers niveaux de l'échelle nous intéressent plus particulièrement, car ils intègrent les publics de fans, qui produisent à leur tour des contenus créatifs à partir des éléments des univers fictionnels. En effet, une partie du public, face aux nouveaux médias, fait ce qu'il convient d'appeler un travail de réappropriation des formes et des contenus, afin de créer des identités culturelles, individuelles et collectives. Celles-ci, fondées sur la (re)production créative, peuvent aussi être considérées comme un dernier avatar de la sérialité. C'est ce dernier aspect que nous aimerions développer, c'est-à-dire quand une production du public vient s'ajouter à celle produite au départ par la franchise et augmenter de fait le phénomène de sérialité.

\section{Du transmedia à l'Histoire}

Le jeu vidéo n'est pas véritablement un point d'entrée des stratégies transmedia des grands groupes et sa qualification comme genre narratif reste toujours discutée ${ }^{9}$. Cependant, comme d'autres types d'œuvres, il peut aussi faire partie des pratiques à travers lesquelles la société est redevable de son univers imaginaire ou de sa perception de la réalité. Il engendre par ailleurs des phénomènes d'extensions fan sur lesquelles nous allons nous attarder. En effet, celles-ci - pour des raisons techniques évidentes comme le fonctionnement à partir du moteur de jeu ou l'usage de logiciels d'éditeurs de niveau fournis par l'éditeur du jeu - restent relativement contraintes et tendent ainsi à s'inscrire dans la sérialité.

Comme en son temps le roman, le jeu vidéo utilise aussi la thématique historique, fabriquant un nouvel imaginaire de l'Histoire chez les joueurs. Si dans le cadre des études vidéoludiques (games studies), très occupées à définir et qualifier leur objet, le sujet n'a pas vraiment encore été approfondi, ce n'est pas le cas chez les historiens (encore avec quelques réserves, car le sujet y est toujours considéré comme une frivolité).

D'une part, ceux-ci sont parfois utilisés comme consultants par les entreprises créatives du secteur. Leur rôle est dans ce cas de légitimer, scientifiquement, non l'ensemble du contenu des jeux, mais les graphismes et le décor. Pour Assassin's Creed Unity, Ubisoft a par exemple recruté comme consultant Jean-Clément Martin, historien, spécialiste de la révolution française et professeur émérite à Paris I, ainsi que Laurent Turcot, professeur à l'université de Trois Rivières (Québec ${ }^{10}$ ). jeu, comme l'article «Histoire et jeux vidéo » de Thomas Rabino ${ }^{11}$. Celui-ci indique :

[...] depuis une petite trentaine d'années, l'histoire au sens large apparait de manière récurrente dans l'univers du jeu vidéo: le passé et ses références communes renforcent le réalisme d'un jeu, donc son potentiel commercial. D'abord restreint à l'utilisation d'un fond historique très flou cantonné aux décors ou à l'allure des personnages pixélisés, l'intégration de scénarii inscrits dans des périodes précises et se prévalant d'une authenticité toute historienne est un phénomène marquant du marché des jeux vidéo. 
19 Parmi les thématiques historiques les plus utilisées par le jeu vidéo, les guerres constituent une part très importante, si ce n'est la plus importante, en particulier la seconde guerre mondiale ${ }^{12}$. une version amateur d'un grand classique de jeu de tir à la première personne (FPS) ayant ce conflit pour théâtre. Nous verrons comment cette extension s'insère dans une problématique esthétique, culturelle et historique très contemporaine et localisée que l'on peut mesurer à partir de son rapport avec la matrice qui a servi à l'engendrer.

21 Les premiers FPS utilisaient principalement la SF comme Doom (ID Software, 1993), ou la fantasy avec Heretic (ID Software, 1994). On note cependant que la seconde guerre mondiale avait inspiré Wolfenstein (ID Software, 1992), mais avec un scénario fantaisiste : il s'agissait de tuer Hitler dans une forteresse truffée de pièges, de soldats et de chiens. Dans les années 2000, ce type de jeu prend un nouveau tournant thématique en utilisant le conflit de 1939-1945, avec un souci de vraisemblance scénaristique et documentaire qui était absent de la plupart des productions vidéoludiques des années 1990. Les sorties de Medal of honor (1999, Dreamworks interactive) et de Call of Duty (2003, Activision) sont deux dates majeures dans l'histoire du genre. Les deux franchises vont utiliser le conflit mondial comme théâtre pour leur jeu, en s'inspirant de l'Histoire et d'œuvres cinématographiques contemporaines à succès comme Il faut sauver le soldat Ryan (Steven Spielberg, 1998) et Stalingrad (JeanJacques Annaud, 2001). Elles produiront plusieurs opus qui deviendront des classiques, Call of duty devenant même le jeu le plus vendu dans le monde en un jour pour les versions de 2009 et de 2014 (7 millions, puis 9,3 millions d'exemplaires). «hollywoodienne", inspirée de nombreux films. Par ailleurs, tout est fait pour immerger au maximum le joueur dans l'ambiance des combats (travail sur le son, sur les décors, sur le graphisme des objets historiques comme les armes). Les missions jouables sont constituées par trois campagnes. La soviétique de 1941-1943 commence à la bataille de Moscou et se termine par la reprise de Stalingrad. La britannique débute en Afrique en 1942 et se poursuit jusqu'au débarquement en 1944. L'Américaine prend la suite au moment du débarquement et s'étend jusqu'à pratiquement la prise de Berlin. Le jeu soigne les références historiques, particulièrement pour les armes que le joueur a la possibilité d'utiliser (mode mono-joueur). Le mimétisme graphique est particulièrement soigné dans ce domaine, car les armes sont les objets que le joueur manipule le plus dans ce genre de jeu. Il révèle aussi un nouveau rapport de la fiction vidéoludique au réel. Si les FPS des années 1990 avaient pour références essentielles la SF, la fantasy ou une Histoire fortement uchronique, ceux des années 2000 montrent indéniablement une préoccupation pour la vraisemblance historique.

À partir de ce grand classique FPS, des fans espagnols vont développer une version originale intitulée España en llamas, qui reste dans l'esprit du jeu puisqu'elle en réutilise le moteur, mais qui porte la guerre sur un terrain que les FPS n'avaient jamais exploré. En effet, la guerre d'Espagne, considérée parfois comme le début de la seconde guerre mondiale, n'a jamais figuré dans les jeux de tir consacrés au conflit.

Sans doute trop exotique pour les studios nord-américains, ce conflit semble être un épisode militaire oublié. Son dénouement est aussi "embarrassant", ou peu euphorique. En 1939, il a consacré le triomphe d'un état fasciste qui n'a disparu 
qu'après la mort de son leader, le général Franco. Par ailleurs, après une période d'isolement, Franco a bénéficié de la guerre froide, qui lui a permis d'obtenir la reconnaissance internationale, l'appui des Etats-Unis et de l'ensemble des pays occidentaux.

Cependant, avant de s'interroger sur les implications socio-historiques du greffon, il convient d'analyser son processus de création. Dans ce cadre, on ne peut compter sur les dossiers de presse. En effet, l'extension possède une première originalité, car elle est l'œuvre d'amateurs passionnés, qui l'ont ensuite diffusée gratuitement. Ils ont financé par la suite l'hébergement de leur forum et du serveur pour le mode multijoueurs grâce à la publicité sur leurs pages. Le développement a duré de 2009 à 2012. Dans notre analyse du résultat, nous ne commenterons pas la dimension technique de la réalisation et les éventuels bugs (erreurs) et nous nous cantonnerons à la version monojoueur.

On peut d'abord souligner le travail de reconstitution historique extrêmement scrupuleux qui a été mis en œuvre ${ }^{13}$. Dans une certaine mesure, le greffon reprend le souci de vraisemblance et de vérisme de la matrice. Les concepteurs ont en effet reproduit les uniformes, les véhicules et les armes d'époque, veillant à ce qu'il n'y ait aucun anachronisme. Ils ont même poussé le souci de la reconstitution en allongeant l'intervalle temporel entre deux tirs pour le joueur quand il utilise le vieux Mauser 1893 qui équipait beaucoup de soldats entre 1936 et 1939 (et qui ne fut pas utilisé pendant la seconde guerre mondiale). L'ensemble a été aussi entièrement sonorisé en espagnol, en utilisant des chansons de la guerre civile enregistrées dans les années trente, qui correspondaient aux deux camps en présence. Le rendu des sons, sans être totalement historique - les auteurs avouent avoir manqué de documentation pour certains engins - n'a pas été négligé, puisque des films d'époque ont servi de base pour fabriquer par exemple le bruit du moteur de l'avion soviétique Polikarpov I16, engin mythique de l'armée républicaine, surnommé familièrement « La mouche » (La Mosca). Enfin, dans les décors urbains, les créateurs ont reproduit sur les murs des affiches de propagande de l'époque. Ils se sont par ailleurs servis des photos du conflit que l'on trouve dans les archives en ligne de l'État pour créer des ambiances graphiques. Au total, sans l'aide d'aucun consultant historien, et avec de fait uniquement les matériaux disponibles sur le Net, l'équipe a essayé de reconstruire une époque.

Suivant le principe de Call of Duty sur lequel repose cette extension, les missions doivent être jouées dans l'ordre. Les concepteurs ont fait alterner les campagnes républicaines et celles des insurgés. Cette alternance répond à la préoccupation pour la vraisemblance historique et respecte la chronologie des batailles proposées. Le joueur, qui sera tour à tour républicain ou insurgé, doit gagner les campagnes qu'il joue. Cellesci correspondent donc à des victoires de chaque camp, qui seront jouées tour à tour, l'empêchant de suivre une voie uchronique, souvent mal vue du point de vue de la rigueur historique.

Les personnages non jouables renvoient principalement à des référents historiques. On retrouve dans ce groupe les généraux fascistes Francisco Franco, Milán Astray, l'officier marocain Mohammed Ben Mizzian ainsi que Wolfram von Richthofen, ou bien les républicains Virgilio Leret (aviateur) et Vicente Rojo (Général). Par contre, tous les personnages jouables sont fictifs. Ce procédé est assez classique dans les genres historiques traditionnels comme le roman où il s'agit de "privilégier les personnages obscurs aux personnalités historiques ${ }^{14} »$. Du côté républicain, c'est une femme, Marión 
Barrena, qui sert d'avatar au joueur pendant presque toutes les missions. Elle renvoie, en la grossissant quelque peu, à la réalité historique de la milicienne anarchiste. Sa présence est complétée par un tankiste russe (Félix Brezhnev) et un médecin anonyme. Dans le camp opposé, l'avatar principal est un soldat des troupes coloniales marocaines Ghanin Shabah, complété par un guardia civil anonyme et Mateo Ferrero, un italien des Corps de Troupes Volontaires (CTV Corpo Truppe Volontarie). Ce dernier finira par déserter et se ranger du côté des Républicains.

Ce casting peut être interprété de deux façons. D'abord, la répartition entre les personnages fictifs et les personnages réels suit la logique des genres littéraires historiques, à savoir que les personnages ayant existé servent principalement à marquer l'immersion dans l'Histoire des personnages fictifs qui sont quant à eux jouables. Comme le fait remarquer Thomas Rabino, pour le jeu vidéo, c'est le désir d'immersion qui pousse aussi à s'éloigner d'un réalisme purement historiographique dont le potentiel ludique est moins grand ${ }^{15}$ :

comment associer l'histoire, dont la quintessence se situe dans l'objectivité, et le jeu vidéo, qui repose sur les actions du joueur, donc sur une subjectivité appelant un cadre fictionnel ? [...] De fait, l'interactivité défait l'histoire et éloigne peu à peu le joueur de la trame établie par la science historique, pour le précipiter dans une variante individualisée et ludique de l'uchronie vue en littérature, au cinéma, et même... en histoire.

On retrouve ici une remarque qu'avait fait Jean-Marie Schaeffer, qui indiquait qu'il fallait distinguer l'immersion, qui visait à brouiller les pistes entre réalité et fiction et la modélisation de la réalité par la fiction (l'utilisation de référents historiques pour créer un jeut).

31 Les avatars du joueur sont donc «décalés " par rapport à la réalité historique et ne correspondent pas exactement aux représentations classiques de la guerre, que ce soit du point de vue historique ou du point de vue fictionnel. Du côté républicain, c'est très flagrant, avec le choix d'une milicienne. Malgré la propagande qui avait été faite sur la participation des volontaires féminines, surtout au début du conflit, elles ne jouèrent qu'un rôle marginal dans les combats. De la même façon, les Russes furent peu nombreux à s'engager dans les batailles, la majorité étant des conseillers techniques et politiques. Les personnages choisis pour jouer les insurgés sont plus représentatifs des combattants, les troupes coloniales, ainsi que le CTV italien représentèrent un effectif nombreux. Mais il faut souligner que le choix se porte sur les deux corps de l'armée qui représentaient le moins les idées politiques de Franco. Il semblerait que les concepteurs, peut-être soucieux de ne pas provoquer de polémiques politiques, aient choisi des personnages dont on ne voit pas immédiatement la dimension idéologique, soit parce qu'ils sont marginaux (le tankiste, la milicienne), soit parce qu'ils sont des «étrangers » au conflit politique. Des miliciens communistes ou anarchistes luttant pour la République, tout comme des phalangistes ou des requetés (corps de troupes monarchistes) soutenant le fascisme auraient renvoyé de façon plus marquée à l'affrontement idéologique qui était l'arrière-plan réel de la guerre. C'est donc le jeu (de guerre) qui prime sur la réalité historique, malgré le souci documentaire affiché par les créateurs, mais qui au bout du compte porte sur le graphisme des objets et du décor et non sur la nature des personnages.

On peut ainsi supposer que le contexte politique espagnol a pesé inconsciemment sur la conception du jeu. Son élaboration se situe dans la continuité d'une période de commémoration du conflit, initiée en 2006. Cette commémoration n'a pas été pour 
l'Espagne un simple épisode de cérémonies. Elle a été l'occasion de rouvrir le dossier de la guerre, que le Franquisme, puis la Transition démocratique avaient soigneusement enterré. En effet, la loi dite de "Mémoire historique ", approuvée par le conseil des ministres de José Luis Rodríguez Zapatero en juillet 2006 et votée définitivement en octobre 2007, reconnaît les droits des victimes de la guerre et du franquisme.

Le jeu opère à sa façon un travail de mémoire qui semble sous-tendue par une préoccupation morale consensuelle. Dans ce cadre, il est intéressant de noter qu'après la fin de la dernière campagne, España en llamas propose une fin alternative ouvrant la voie à l'uchronie: les Républicains gagnent la guerre et capturent Franco. Malgré le désir " d'équilibre » et la préoccupation historique, le désir de faire triompher le bon camp transparaît. Comme pour les chansons de la guerre, que nous avons abordées dans un autre travail ${ }^{17}$, la mémoire s'est faite sélective, malgré les contraintes historicistes du jeu. Alors qu'ils exposent dans leur forum une somme considérable de documents historiques, les fans créateurs ont opté pour une fin alternative clairement en rupture avec l'Histoire mais moralement meilleure au regard de l'époque contemporaine.

\section{Conclusion}

La création transmédia fait émerger une nouvelle conception de la sérialité, qui se décline sur plusieurs modes. Pour les productions classiques de franchise, on peut faire le même constat que Jean-Marie Schaeffer ${ }^{18}$ :

Il s'agit d'arts technologiques, [qui] dépendent en partie d'un savoir-faire partagé et nécessitent une exécution parfois collective, en plusieurs étapes planifiées ; enfin ils ont souvent besoin de financements importants et ne sauraient échapper à la question de la rentabilité économique. Ils permettent donc à l'argument antimimétique de s'appuyer sur les idéaux ségrégationnistes (l'art contre la technique) et anti-économique (l'art contre l'industrie des médias) qui commandent en grande partie la manière dont nous pensons actuellement les relations entre l'art et la société. [...] Cette rupture est censée correspondre à une différence de statut : l'art d'un côté, l'industrie (et le commerce de l'autre).

Cependant, on voit avec les créations de fans, sans intention lucrative majeure, que ces fictions peuvent aussi échapper à l'objectif commercial pour en remplir d'autres. On se retrouve alors en décalage avec les sérialités d'intention commerciale. La relecture qu'opèrent les fans espagnols d'un classique FPS est ainsi intéressante à plus d'un titre.

En ce qui concerne l'exemple que nous avons analysé, nous avons perçu plusieurs paradoxes. L'Histoire, comme l'indique Thomas Rabino « rend plus dense la fiction du jeu $^{19}$ ", surtout pour des espagnols, en rendant le jeu de tir plus «local», corrigeant ainsi le défaut de la majorité des FPS historiques produits à partir du point de vue américain qui se fondent généralement sur une culture transnationale. Bien que l'entreprise soit le fait d'amateurs, elle a été accueillie sur la page de la franchise, aux côtés d'autres mods qui mettent en scène par exemple la guerre en Yougoslavie ${ }^{20}$.

Comme le cinéma ou le roman historique, le jeu vidéo historique se charge d'une idéologie et de représentations du passé qui reflètent l'imaginaire collectif ou le roman national de son époque. España en llamas nous révèle un désir de mettre les deux camps à égalité et de gommer ainsi à l'avance toute polémique idéologique sur la guerre. Pour le fan créatif, « l'intérêt est bien [...] de faire sens pour soi et son monde, en articulant ce sens à ses affects ${ }^{21}$ ", qui, dans le cas de ce mod, a une portée nationale. 


\section{NOTES}

1. Schaeffer, Jean-Marie, Pourquoi la fiction, Paris, Seuil, 1999, p. 11.

2. Ce n'est pas toujours le cas, dans la série d'enquêtes de Gabriel Lecouvreur dit le Poulpe (Gallimard), les romans sont écrits par une pléiade d'auteurs français.

3. Henry Jenkins, La culture de la convergence, Paris, Armand Colin, 2013, p. 155.

4. Jenkins, Op. cit., p. 156.

5. Jenkins, Op. cit., p. 157.

6. Jenkins, Op. cit., p. 113.

7. Jenkins, art. cit. pp. 149-150.

8. Céline Masoni-Lacroix, "Le transmédia, terrain d'acculturation communicationnelle des publics? Vers une approche narratologique communicationnelle " in Le storytelling, succès des histoires, histoire d'un succès, Paris, L'Harmattan, 2012, p. 59.

9. Voir à ce propos Marc Marti et Raphaël Baroni, Les bifucations du récit interactif, continuités ou ruptures?, Cahiers de Narratologie, 27 | 2014, mis en ligne le 19 décembre 2014, consulté le 04 février 2016. URL : http://narratologie.revues.org/7077

10. Lebrun, Jean, « Assassin's Creed Unity, un jeu vidéo sur la Révolution française ", La marche de l'Histoire, 9 décembre 2014, France Inter, URL : http://www.franceinter.fr/emission-la-marchede-lhistoire-assassin-s-creed-unity-un-jeu-video-sur-la-revolution-francaise

11. Rabino, Thomas, « Jeux vidéo et Histoire », Le Débat, 2013/5, n 177, p. 110-116.

12. Rabino, art. cit. p. 113.

13. Pour tout ce qui suit, nous renvoyons aux détails fournis par l'équipe de développement sur son forum. http://1936.forogratis.es/portal.php

14. Deruelle, Aude, Tassel, Alain (éds.), Problèmes du roman historique, Paris, L'Harmattan, 2008, p. 7.

15. Rabino, art. cit. p. 114.

16. Jean-Marie Schaeffer, Op. cit., p. 39.

17. Marc Marti, « La mémoire multimédia, les chansons de la guerre civile sur Youtube » in Karim Benmiloud, Florence Belmonte, Sylvie Imparato Prieur, Guerres dans le monde ibérique et ibéroaméricain, Peter Lang, 2014, pp. 531-539.

18. Jean-Marie Schaeffer, Op. cit. p. 26.

19. Rabino, art. cit., p. 116.

20. http://callofduty.filefront.com/files/Call_of_Duty_2/Mods/Total_Conversions;8580

21. Masoni-Lacroix, art. cit., p. 83.

\section{ABSTRACTS}

L'article propose de questionner la notion de sérialité non seulement à partir de sa valeur esthétique, mais aussi à partir des fonctions sociales qu'elle en vient à assumer, car la sérialité est tout autant un mode de production des objets culturels qu'un mode de réception/consommation. Nous insisterons sur le fait que la réception "peut mettre en série » des objets qui au départ n'avaient pas été conçus dans cette optique. Ainsi, les nouvelles formes de la culture 
contemporaine ont fait émerger un autre type de sérialité, celle d'une déclinaison narrative dans le transmedia storytelling, qui place le spectateur dans une position créative, ou du moins dans un mode de consommation dynamique et non linéaire. Afin d'illustrer cette logique, nous analyserons la relation qui s'établit entre le public et la série, en examinant quelle façon celle-ci peut conditionner une fan fiction, constituée dans le cadre de notre contribution par un greffon (mod) de jeu vidéo, intitulé España en llamas et qui repose sur le FPS classique Call of Duty 2 (2005).

El artículo propone analizar la noción de serialidad no sólo a partir de su valor estético sino también interrogando sus funciones sociales, siendo la serialidad tanto un modo de producción como un modo de consumo/recepción de las obras. Insistiremos en el hecho de que la recepción puede "crear series", a partir de objetos que básicamente no se destinaban a ese tipo de uso. Así, en las nuevas formas de la cultura contemporánea, emerge otro tipo de serialidad, que consiste en una declinación narrativa por el transmedia storytelling, que otorga al espectador un papel creador, o, por lo menos, en una posición de consumidor activo. Para ilustrar esa nueva lógica, analizaremos la relación que se establece entre el público y una serie, examinando de que manera puede condicionar una fan fiction (ficción aficionado). En este artículo se estudia el caso de un mod del juego vídeo Call of Duty 2 titulado España en llamas.

\section{INDEX}

\section{Geographical index: Espagne}

Chronological index: XXIe siècle

Mots-clés: guerre civile espagnole, jeu vidéo, narration, greffon, sérialité

\section{AUTHOR}

\section{MARC MARTI}

Université Nice Sophia Antipolis, LIRCES EA3159 